\section{SP0007 OSTEOARTHRITIS: NEW CONCEPTS OF PATHOGENESIS AND MANAGEMENT GUIDELINES}

RW Moskowitz. Case Western Reserve University, University Hospitals of Cleveland, Cleveland, $\mathrm{OH}$, USA

10.1136/annrheumdis-2001.28

Osteoarthritis (OA), the most common form of arthritis, is increasing in prevalence as the population ages. Osteoarthritic pathology occurs as a result of an imbalance between synthesis and repair (catabolic) processes. Synthesis, increased early in the osteoarthritic process, cannot keep pace with catabolic processes in which proteolytic and collagenolytic enzymes destroy cartilage matrix. Inflammation, the result of an interplay between cartilage breakdown and synovial reaction, increases in severity as the disease progresses.

Current concepts suggest a specific role for the ageing process wherein formation of Advanced Glycation End-products (AGEs) increase with age, resulting in increased cross-linking of cartilage matrix components. AGEs also activate cytokines, adding to the inflammatory reaction.

Genetic defects in type II collagen have been described. The most common mutation demonstrated is an error in one base leading to a substitution of cysteine for arginine in the collagen matrix, resulting in generalised precocious familial OA. Additional mutations have been described in other sites in type II collagen.

Pain in OA results from multifactorial interplays, including inflammatory and non-inflammatory factors. Management is targeted to treating pain, improving function and disease modification. Symptomatic therapy includes simple analgesics, Cox-2 selective non-steroidal anti- inflammatory agents, traditional non-steroidal anti-inflammatory agents (with or without gastropathy-protective agents), and, at times, opiates. Cox-2 non-steroidal anti-inflammatory agents represent a significant advance in therapy, based on decreased peptic-ulcer-related gastropathy including haemorrhage, perforation or obstruction. Intra-articular steroids are helpful for acute flares. Intra-articular hyaluronans, an additional new modality, leads to relief of pain in OA of the knee in a significant number of patients; pain is relieved for prolonged periods of time, and therapy with repeated cycles is safe and effective. Hyaluronans are of particular benefit in patients in whom non-steroidal anti-inflammatory agents are not effective or well-tolerated.

Nutraceuticals such as glucosamine and chondroitin sulfate have been shown to relieve pain in a number of studies, and, in some recent investigations, to modify the basic disease process.

Agents which inhibit enzymatic or cytokine-induced breakdown and/or which stimulate matrix synthesis may lead to structure modification. Demonstration of such modification is difficult, and studies require several years duration. A number of agents appear to be disease - modifying in animal models, and successful disease-modification in humans is now considered tenable. Replacement of cartilage with autologous chondrocytes, or mesenchymal bone marrow stem cells shows promise for direct cartilage repair.

\section{REFERENCES}

1 Altman RD, Hochberg MD, Moskowitz RW, Schnitzer TJ. Recommendations for the medical management of osteoarthritis of the hip and knee: 2000 update. Arthritis Rheum. 2000;43:1905-15

2 Crofford LJ, Lipsky PE, Brooks P, Abramson SB, Simon LS, vandePutte LBA. Basic biology and clinical application of specific cycloxygenase-2 inhibitors. Arthritis Rheum. 2000;43:4-13
3 Altman RD, Moskowitz RW. Intra-articular sodium hyaluronate (Hyalgan) in the treatment of patients with osteoarthritis of the knee: a randomized clinical trial. $J$ Rheumatol. 1998;25:2203-12

4 Deal CL, Moskowitz RW. Nutraceuticals as therapeutic agents in osteoarthritis. Rheum Dis Clin North Am. 1999;25:379-95

\section{Activity related diseases - Friday 15 June, 16.00-17.30/Congress Hall}

\section{SP0077 INNATE VERSUS ADAPTIVE IMMUNITY IN THE PATHOGENESIS OF ARTHRITIS}

R Holmdahl. Medical Inflammation Research, Lund University, Lund, Sweden

10.1136/annrheumdis-2001.29

Despite large efforts, the genetic and environmental factors that cause rheumatoid arthritis (RA) have not been found. The only significant genetic clue today is an association with the major histocompatibility complex (MHC) region coding for class II peptide receptors, which are known to be crucial for immune responses. However, the MHC region only partially reflects the genetic influence as this is a polygenically controlled disease. In addition environmental factors are likely to strongly influence the disease. One such important environmental factors are adjuvants, derived from pathogenic organism or from other exogenous and endogenous sources.

These complex issues are better clarified in animal models. In fact there are mainly two ways to induce arthritis in rodents.

Firstly, injection of various adjuvants trigger arthritis in certain rat strains. ${ }^{1,2,3}$ The properties of these adjuvants are that they are difficult to degrade in vivo and are soluble in cellular membranes. In contrast to antigens they do not bind to MHC molecules and can therefore not elicit an antigen specific response. Nevertheless, adjuvants trigger an arthritic disease which in certain cases closely mimics RA(3). One of the best models is pristine induced arthritis (PIA) which is a MHC class II dependent, ab T cell dependent chronic relapsing disease. ${ }^{3,4,5}$ It can be prophylactically and therapeutically vaccinated against with the help of joint specific antigens. ${ }^{6}$

Secondly, immunisation with joint-specific proteins may induce arthritis. In this case fragments of the protein bind to MHC molecules and elicit an immune response cross-reactive with joint antigens. ${ }^{7,8,9}$ Subsequently the joints are attacked and may develop into a chronic relapsing disease depending on the specificity of the immune response and the genetics of the strain. Examples of joint antigen specific models are type II collagen induced arthritis (CIA) and cartilage oligomeric matrix proteininduced arthritis (COMPIA)..$^{10,11,12}$

Genetic analyses of adjuvant and joint specific models show that most, but not all, genetic regions are shared indicating that they share pathogenic mechanisms. These models will be further discussed mainly based on genetic dissection of MHC and nonMHC genetic regions.

\section{REFERENCES}

1 Pearson CM. Development of arthritis, periarthritis and periostitis in rats given adjuvants. Proc Soc Exp Biol Med. 1956;91:95

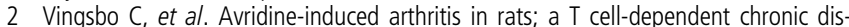
ease influenced both by MHC genes and by non-MHC genes. Clin Exp Immunol. 1995:99:359

3 Vingsbo $C$, et al. Pristane-induced arthritis in rats: a new model for rheumatoid arthritis with a chronic disease course influenced by both major histocompatibility 